\title{
Limits to the muon flux from WIMP annihilation in the center of the Earth with the AMANDA detector
}

J. Ahrens, ${ }^{1}$ E. Andrés, ${ }^{2}$ X. Bai, ${ }^{3}$ G. Barouch,${ }^{4}$ S. W. Barwick, ${ }^{5}$ R. C. Bay, ${ }^{6}$ T. Becka, ${ }^{1}$ K.-H. Becker, ${ }^{1}$ D. Bertrand, ${ }^{7}$ A. Biron, ${ }^{8}$ O. Botner, ${ }^{9}$ A. Bouchta ${ }^{8, *}$ S. Carius,${ }^{10}$ A. Chen, ${ }^{4}$ D. Chirkin, ${ }^{6,11}$ J. Conrad, ${ }^{9}$ J. Cooley, ${ }^{4}$ C. G. S. Costa, ${ }^{7}$ D. F. Cowen, ${ }^{12}$ E. Dalberg, ${ }^{2, \dagger}$ C. De Clercq, ${ }^{13}$ T. DeYoung, ${ }^{4, \$}$ P. Desiati, ${ }^{8}$ J.-P. Dewulf, ${ }^{7}$ P. Doksus, ${ }^{4}$ J. Edsjö, ${ }^{2}$

P. Ekström, ${ }^{2}$ T. Feser, ${ }^{1}$ T. K. Gaisser, ${ }^{3}$ M. Gaug, ${ }^{8,8}$ L. Gerhardt, ${ }^{5}$ A. Goldschmidt, ${ }^{14}$ A. Goobar, ${ }^{2}$ A. Hallgren, ${ }^{9}$ F. Halzen, ${ }^{4}$ K. Hanson, ${ }^{12}$ R. Hardtke, ${ }^{4}$ T. Hauschildt, ${ }^{8}$ M. Hellwig, ${ }^{1}$ G. C. Hill, ${ }^{4}$ P. O. Hulth, ${ }^{2}$ S. Hundertmark, ${ }^{5}$ J. Jacobsen, ${ }^{14}$ A. Karle, ${ }^{4}$ J. Kim,${ }^{5}$ B. Koci, ${ }^{4}$ L. Köpke, ${ }^{1}$ M. Kowalski,${ }^{8}$ J. I. Lamoureux,${ }^{14}$ H. Leich, ${ }^{8}$ M. Leuthold ${ }^{8}$ P. Lindahl,${ }^{10}$ P. Loaiza, ${ }^{9}$ D. M. Lowder, ${ }^{6, \|}$ J. Ludvig, ${ }^{14}$ J. Madsen ${ }^{4}$ P. Marciniewski, ${ }^{9, \pi}$ H. S. Matis,${ }^{14}$ C. P. McParland, ${ }^{8}$ T. C. Miller, ${ }^{3, * *}$

Y. Minaeva, ${ }^{2}$ P. Miočinović, ${ }^{6}$ P. C. Mock,${ }^{5, \dagger}$ R. Morse, ${ }^{4}$ T. Neunhöffer ${ }^{1}$ P. Niessen, ${ }^{13}$ D. R. Nygren, ${ }^{14}$ H. Ogelman, ${ }^{4}$ Ph. Olbrechts, ${ }^{13}$ C. Pérez de los Heros,,${ }^{9}$ A. Pohl,${ }^{10}$ R. Porrata, ${ }^{5,8}$ P. B. Price, ${ }^{6}$ G.T. Przybylski, ${ }^{14}$ K. Rawlins, ${ }^{4}$ W. Rhode,${ }^{11}$

M. Ribordy, ${ }^{8}$ S. Richter, ${ }^{4}$ J. Rodríguez Martino, ${ }^{2}$ P. Romenesko, ${ }^{4}$ D. Ross, ${ }^{5}$ H.-G. Sander, ${ }^{1}$ T. Schmidt,${ }^{8}$ D. Schneider, ${ }^{4}$

E. Schneider ${ }^{5}$ R. Schwarz, ${ }^{4}$ A. Silvestri, ${ }^{11,8}$ M. Solarz,${ }^{6}$ G. M. Spiczak, ${ }^{15}$ C. Spiering, ${ }^{8}$ D. Steele, ${ }^{4}$ P. Steffen, ${ }^{8}$

R. G. Stokstad, ${ }^{14}$ O. Streicher, ${ }^{8}$ P. Sudhoff, ${ }^{8}$ K. H. Sulanke, ${ }^{8}$ I. Taboada, ${ }^{12}$ L. Thollander, ${ }^{2}$ T. Thon, ${ }^{8}$ S. Tilav, ${ }^{3}$ M. Vander Donckt, ${ }^{7}$ C. Walck, ${ }^{2}$ C. Weinheimer, ${ }^{1}$ C. H. Wiebusch, ${ }^{8, *}$ C. Wiedemann, ${ }^{2}$ R. Wischnewski, ${ }^{8}$

H. Wissing, ${ }^{8}$ K. Woschnagg, ${ }^{6}$ W. Wu, ${ }^{5}$ G. Yodh, ${ }^{5}$ and S. Young ${ }^{5}$

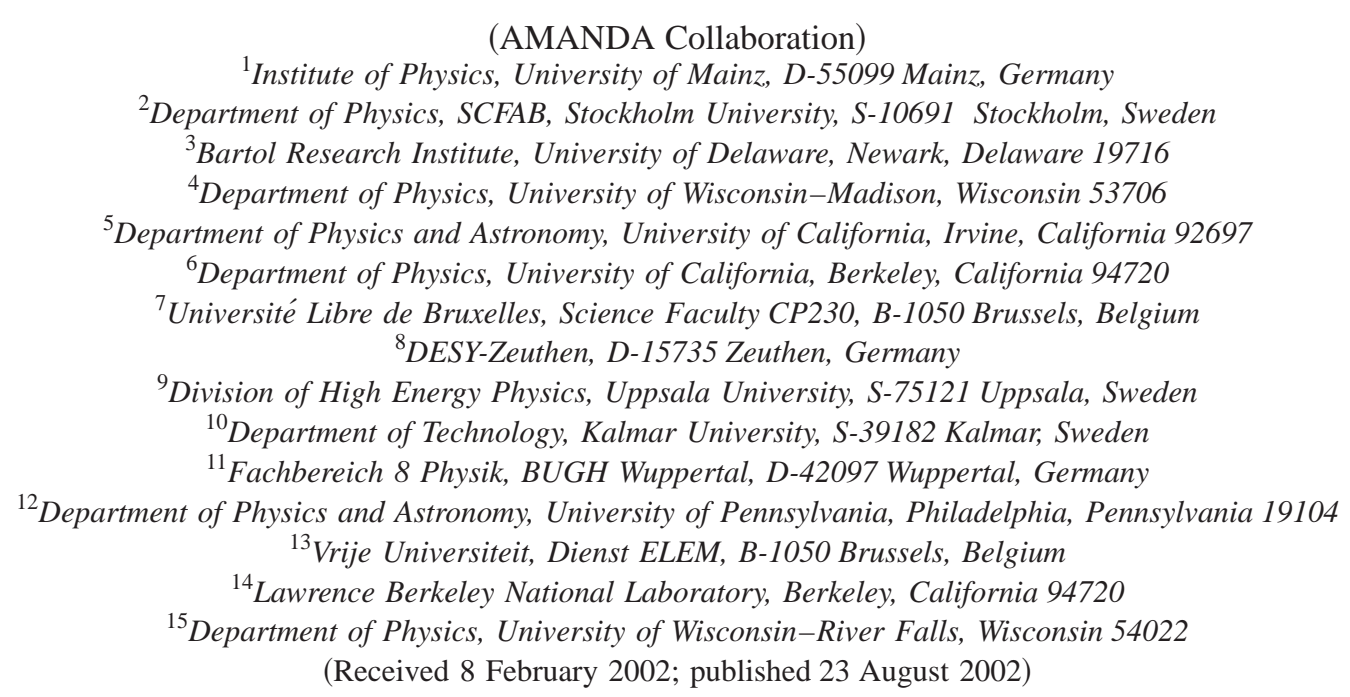

A search for nearly vertical up-going muon-neutrinos from neutralino annihilations in the center of the Earth has been performed with the AMANDA-B10 neutrino detector. The data collected in 130.1 days of live time in 1997, $\sim 10^{9}$ events, have been analyzed for this search. No excess over the expected atmospheric neutrino background has been observed. An upper limit at $90 \%$ confidence level has been obtained on the annihilation rate of neutralinos in the center of the Earth, as well as the corresponding muon flux limit, both as a function of the neutralino mass in the range $100 \mathrm{GeV}-5000 \mathrm{GeV}$.

DOI: $10.1103 /$ PhysRevD.66.032006

PACS number(s): 95.35.+d, 11.30.Pb, 95.30.Cq

\footnotetext{
*Currently at CERN, CH-1211, Genève 23, Switzerland.

${ }^{\dagger}$ Currently at Defense Research Establishment (FOA), S-17290 Stockholm, Sweden.

*Currently at Santa Cruz Institute for Particle Physics, University of California-Santa Cruz, Santa Cruz, CA 95064.

${ }^{\S}$ Currently at IFAE, 08193 Barcelona, Spain.

${ }^{\|}$Currently at MontaVista Software, 1237 E. Arques Ave., Sunnyvale, CA 94085.

${ }^{\pi}$ Currently at The Svedberg Laboratory, S-75121, Uppsala, Sweden.

**Currently at Johns Hopkins University, Applied Physics Laboratory, Laurel, MD 20723.

${ }^{\dagger}$ Currently at Optical Networks Research, JDS Uniphase, 100 Willowbrook Rd., Freehold, NJ 07728-2879.

\$ Corresponding author. E-mail: cph@tsl.uu.se

${ }^{\S}$ Currently at L-174, Lawrence Livermore National Laboratory, 7000 East Ave., Livermore, CA 94550.
} 


\section{INTRODUCTION}

There are strong observational indications for the existence of dark matter in the universe. Measurements of the energy density of the universe, $\Omega_{0}$, from the combined analysis of cosmic microwave background radiation data and high redshift type Ia supernovae favor $\Omega_{0}=1$, with a matter $\Omega_{\mathrm{M}}$ and a cosmological constant $\Omega_{\Lambda}$ component. Combined with data from rotation curves of galaxies and cluster mass measurements, the matter contribution to $\Omega_{0}$ is $0.3 \leqslant \Omega_{\mathrm{M}}$ $\leqslant 0.4$. Big bang nucleosynthesis calculations of primordial helium, lithium and deuterium production, supported by abundance measurements of these elements, set an upper limit on the amount of baryonic matter that can exist in the universe, $\Omega_{\mathrm{B}} \leqslant 0.05$ (see Ref. [1] for a recent review of values of $\Omega$ ). Non-baryonic dark matter must therefore constitute a substantial fraction of $\Omega_{\mathrm{M}}$.

In this paper we present results of a search for nonbaryonic dark matter in the form of weakly interacting massive particles (WIMP) using the Antartic Muon and Neutrino Detector Array (AMANDA) high-energy neutrino detector. Section II contains a brief motivation for WIMPs as dark matter candidates. Section III describes the characteristics of the AMANDA detector in the configuration used for this analysis. Sections IV and V contain a description of the simulation and analysis techniques used. In Sec. VI we discuss the sources of the current systematic uncertainties of our analysis. In Sec. VII we present the results of the analysis and we introduce a novel way of calculating upper limits in the presence of systematic uncertainties. An upper limit on the neutrino-induced muon flux expected from WIMP annihilation in the center of the Earth is derived with this method. A comparison with published muon-flux limits obtained by existing neutrino experiments is presented in Sec. VIII.

\section{WIMPS AS DARK MATTER CANDIDATES}

Particle physics provides an interesting dark matter candidate as a weakly interacting massive particle (WIMP). The relic density of particle type $i$ depends on its annihilation cross section, $\sigma$, as $\Omega_{i} h^{2} \sim 3 \times 10^{-27} /\langle\sigma \mathrm{v}\rangle$ (neglecting massdependent logarithmic corrections), where \langle\rangle indicates thermal average and $\mathrm{v}$ is the relative velocity of the particles involved in the collision (see, for example, Ref. [2]). Weak interactions provide the right annihilation cross section for the WIMPs to decouple in the early universe and give a relic density within the required range to contribute substantially to the energy density of the universe today. This is basically what would be needed to solve the dark matter problem.

In particular, and starting from a completely different rationale, the minimal supersymmetric extension to the standard model of particle physics (MSSM) provides a promising WIMP candidate in the neutralino, $\chi$. The neutralino is a linear combination of the $B$-ino $\widetilde{B}$ and the $W$-ino $\widetilde{W}$, the supersymmetric partners of the electroweak gauge bosons, and of the $\mathrm{H}_{1}^{0}$ and $\mathrm{H}_{2}^{0}$, the neutral Higgs bosons, and it is stable (assuming $R$-parity conservation, which is further supported to avoid too rapid proton decay). The actual compo- sition of the neutralino can have cosmological consequences since its annihilation cross section depends on it. For example, it has been argued that a mainly $W$-ino type neutralino would not be cosmologically relevant in the present epoch since it would have annihilated too fast in the early universe to leave any relevant relic density [3].

Still, the large parameter space of minimal supersymmetry can be exploited to build realistic models which provide relic neutralino densities within the cosmologically interesting region of $0.025 \leqq \Omega_{\chi} h^{2}<1$. Negative results from searches for supersymmetry at the LEP accelerator at CERN have set a lower limit on the neutralino mass $m_{\chi}>31 \mathrm{GeV}$ (Ref. [4]), while theoretical arguments based on the requirement of unitarity set an upper limit of $340 \mathrm{TeV}$ (Ref. [3]). Imposing in addition the condition on $\Omega_{\chi} h^{2}$ mentioned above, only models with $m_{\chi} \lesssim 10 \mathrm{TeV}$ (Ref. [5]) become cosmologically interesting.

Neutralinos have a non-negligible probability of scattering off nuclei of ordinary matter. Assuming the dark matter in the Galactic halo is (at least partially) composed of relic neutralinos, elastic interactions of these particles with nuclei in the Earth can lead to energy losses that bring the neutralino below the escape velocity, becoming gravitationally trapped [6,7]. For high neutralino masses (greater than a few hundred $\mathrm{GeV}$ ) direct capture from the halo population by the Earth is kinematically suppressed [8]. In this case neutralinos can be accreted from the population already captured by the solar system. Gravitational capture is expected to result in an accumulation of neutralinos around the core of the Earth, where they will annihilate. An equilibrium density is reached when the capture rate equals the annihilation rate. Neutrinos are produced in the decays of the resulting particles, with an energy spectrum extending over a wide range of values and bounded from above by the neutralino mass. Annihilation of neutralinos directly into neutrinos (or light fermion pairs in general) is suppressed by a factor $m_{\mathrm{f}}^{2} / m_{\chi}^{2}$ due to helicity constraints, where $m_{\mathrm{f}}$ is the fermion mass. Neutrino detectors can therefore be used to constrain the parameter space of supersymmetry by setting limits on the flux of neutrinos from the center of the Earth [2,9]. Note that this indirect neutralino detection will be favored for high neutralino masses, since the cross section of the resulting neutrinos with ordinary matter scales with $E_{\nu}$.

\section{THE AMANDA-B10 DETECTOR}

The AMANDA-B10 detector consists of an array of 302 optical modules deployed in ten vertical strings at depths between $1500 \mathrm{~m}$ and $2000 \mathrm{~m}$ in the South Pole ice cap. The strings are arranged in two concentric circles of $60 \mathrm{~m}$ and $120 \mathrm{~m}$ diameter, respectively. The modules on the four inner strings are separated by $20 \mathrm{~m}$ in the vertical direction, while in the outer six strings the vertical separation between modules is $10 \mathrm{~m}$. An optical module consists of a photomultiplier tube housed in a spherical glass pressure vessel. Coaxial cables (in the inner four strings) and twisted quad cables (in the outer six strings) provide the high voltage to the photomultiplier tubes and transmit the signals to the data acquisition electronics at the surface. 
Muons from charged-current high-energy neutrino interactions near the array are detected by the Cherenkov light they produce when traversing the ice. The relative timing of the Cherenkov photons reaching the optical modules allows the reconstruction of the muon track. A more detailed description of the detector is given in Ref. [10]. The detector was triggered when a majority requirement was satisfied: an event was recorded if at least 16 modules had a signal within a predefined time window of $2 \mu \mathrm{s}$. The data taking rate was $100 \mathrm{~Hz}$.

AMANDA-B10 was in operation during the 1997 Antarctic winter. The separation of 300 atmospheric neutrinos from the data sample collected in that period established the detector as a high-energy neutrino telescope [11]. The array was upgraded with 122 more modules during the antarctic summer 1997-1998 and in 1999-2000 253 additional ones were added, completing the proposed design of 677 optical modules in 19 strings, AMANDA-II [12].

\section{SIGNAL AND BACKGROUND SIMULATIONS}

\section{A. Simulation of neutralino annihilations}

Neutralinos can annihilate pairwise to, e.g., $l^{+} l^{-}, q \bar{q}$, $W^{+} W^{-}, Z^{0} Z^{0}, H_{1,2}^{0} H_{3}^{0}, Z^{0} H_{1,2}^{0}$ and $W^{ \pm} H^{\mp}$. Neutrinos are produced in the decays of these annihilation products. Neutrinos produced in quark jets (from e.g. $b \bar{b}$ or Higgs bosons) typically have lower energy than those produced from decays of $\tau$ leptons and gauge bosons. We will refer to the first type of annihilation channels as "soft" and to the second as "hard."

The simulations of the expected neutralino signal were done in the framework of the SUSY models described in Ref [13]. The hadronization and decay of the annihilation products have been simulated using PYTHIA [14]. The simulations were performed for six different WIMP masses between 10 $\mathrm{GeV}$ and $5000 \mathrm{GeV}$. For each mass, six different annihilation channels $\left(c \bar{c}, b \bar{b}, t \bar{t}, \tau^{+} \tau^{-}, W^{+} W^{-}\right.$and $\left.Z^{0} Z^{0}\right)$ were considered, with $1.25 \times 10^{6}$ events generated for each. Note that the decay of $b$ and $c$ hadrons will take place in matter instead of vacuum. This was incorporated in the simulations in an effective manner justified by the fact that, for the neutralino masses considered, the reinteractions of these heavy hadrons with the surrounding medium are not dominant, and can be parametrized as an effective energy loss at the time of decay. As a reference soft spectrum, we chose the annihilation into $\mathrm{b} \bar{b}$, and as a reference hard spectrum, the annihilation into $W^{+} W^{-}$. For a given mass, these two spectra can be regarded as extreme cases. We have used these channels in the analysis described below, bearing in mind that a typical spectrum would lie somewhere in between.

\section{B. Simulation of the atmospheric neutrino flux}

Neutrinos from the decay of secondaries produced in cosmic ray interactions in the atmosphere constitute the physical background to the neutralino search. We have simulated this atmospheric neutrino flux using the calculations of Lipari [15]. To obtain the rate of neutrino interactions producing muons we have used the neutrino and anti-neutrino-nucleon cross sections from Gandhi et al. [16]. The actual neutrinonucleon interactions have been simulated with PYTHIA using the CTEQ3 [17] parametrization of the nucleon structure functions. The use of PYTHIA allows to model the hadronic shower produced at the vertex of the interaction and, therefore, to calculate the Cherenkov light produced by secondaries. When the neutrino-nucleon interaction occurs within the instrumented volume of the detector, this is a non-negligible contribution to the total event light output.

A three-year equivalent atmospheric neutrino sample with energies between $10 \mathrm{GeV}$ and $10 \mathrm{TeV}$ and zenith angles between $90^{\circ}$ (horizontal) and $180^{\circ}$ (vertically up-going) has been simulated [18]. The sample contains $3.7 \times 10^{7}$ events, of which 41234 triggered the detector.

\section{Simulation of the atmospheric muon flux}

The majority of the triggers in AMANDA are induced by muons produced in cosmic ray interactions in the atmosphere and reaching the detector depth. The simulation of this atmospheric muon flux was performed using the BASIEV [19] program. We note that this program only uses protons as primaries. However, the systematic uncertainty introduced by this approximation is negligible in comparison with that from the present uncertainty in the primary flux intensity. Moreover, heavier nuclear primaries produce more muons per interaction, but with lower energies on average [20], which will in general loose all their energy and decay before reaching the detector. A study performed using the CORSIKA [21] air shower generator, with the QGSJET option to model the hadronic interactions, including the complete cosmic ray composition confirms this scenario.

The simulation of a statistically significant sample of atmospheric muon background is an extremely high CPU-time consuming task due to the strong rejection factors needed. We have simulated $6.3 \times 10^{10}$ primary interactions, distributed isotropically with zenith angles, $\Theta$, between 0 and 85 degrees, and with energies, $E$, between $1.3 \mathrm{TeV}$ and 1000 $\mathrm{TeV}$, assuming a differential energy distribution $\propto E^{-2.7}$ (Ref. [22]). The total number of triggers produced were $5 \times 10^{6}$. Normalizing to the primary cosmic ray rate, the generated sample corresponds to about 0.6 days of equivalent detector live-time. Due to the narrow vertical angular cones used for this analysis this background sample is sufficient to model the detector response and develop the rejection cuts. In addition, a larger sample of background data was used in the training of the discriminant analysis program used as cut level 4. This is described in more detail in the next section.

\section{Muon propagation}

The muons produced in the signal and background simulations described above were propagated from the production point to the detector taking into account energy losses by bremsstrahlung, pair production, photo-nuclear interactions and $\delta$-ray production from Ref. [23]. The Cherenkov light emitted by the secondaries produced in these processes is taken into account when calculating the response of the detector to the passage of the muon. 


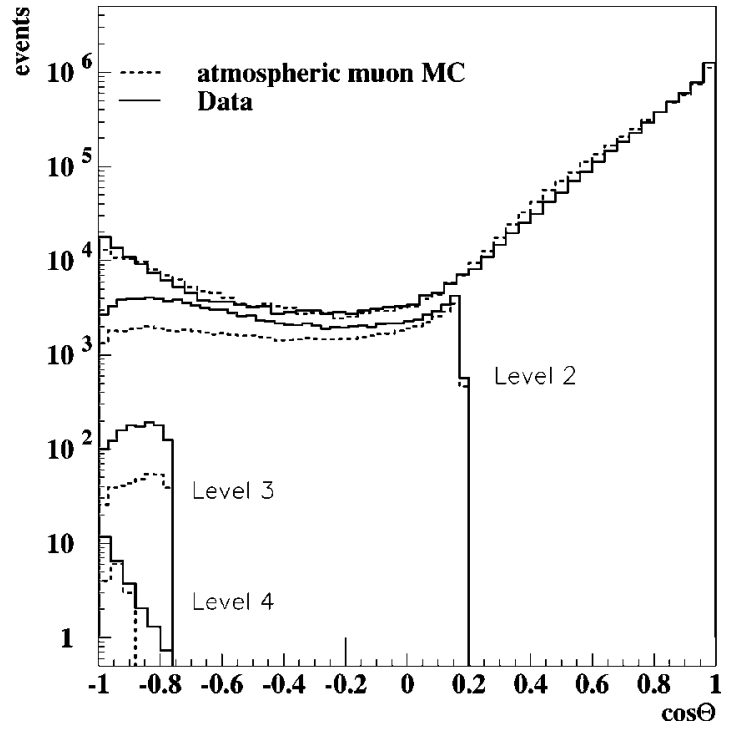

FIG. 1. Angular distributions of data and atmospheric muon simulation Monte Carlo (MC) at different analysis levels. Top to bottom: trigger to level 4 . The distributions are normalized to the simulated sample, $5 \times 10^{6}$ events.

\section{DATA ANALYSIS}

The analysis presented in this paper was performed on data taken with the 10-string AMANDA detector between March and November 1997. The experimental data set consists of $1.05 \times 10^{9}$ events in a total of 130.1 days of detector live-time. The data were first cleaned of noise hits and hits from optical modules that were unstable during the running period. Short pulses that are likely induced by cross talk between channels are also rejected at this stage. Details on the data cleaning procedure are given in Ref. [24]. The data are then reconstructed and five filters consisting of cuts based on the event hit pattern and the quality of the reconstruction are applied in order to identify potential up-going neutrino candidates. The distributions of the reconstructed zenith angle from trigger level (after hit cleaning) until filter level 4 for data and simulated atmospheric muons are shown in Fig. 1. The curves have been normalized to the simulated sample, $5 \times 10^{6}$ events. The uppermost curves in the plot show the reconstructed direction without any quality criteria applied to the fits, showing good agreement between the data and the Monte Carlo sample along the whole angular range. The curves clearly indicate that a small percentage (about 2\%) of the originally down-going tracks are misreconstructed as upgoing ( $\cos \Theta$ less than zero the figure). The series of cuts described below were developed to reject such misreconstructions, and their effect on the angular distribution is also shown in Fig. 1 for comparison. The filter level 2 and level 3 curves show that the filtering procedure is more effective rejecting the simulated muon background than the data. This is due to detector effects not included in the simulation of the detector response and surviving to these levels, like electronic cross talk between channels or inefficiencies of the digitizing electronics. Other processes not included in the background simulations that can contribute to the discrepancy are overlapping events from uncorrelated cosmic ray

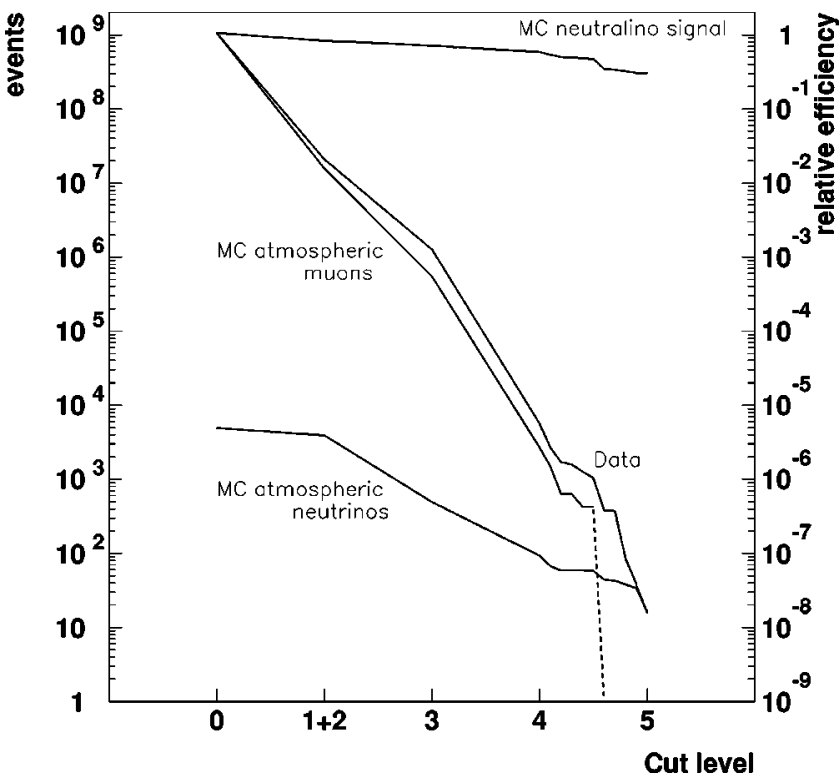

FIG. 2. Rejection and efficiency at each filter level for the data and simulations of the neutralino signal, atmospheric neutrinos and atmospheric muons. The dashed part corresponds to rejection levels surpassing the statistical precision of the simulated sample, yielding zero remaining events. The neutralino signal curve should be read only with respect to the right axis scale, and it shows the relative signal efficiency with respect to trigger level. The rest of the curves are plotted with respect to the left axis scale.

interactions and the contribution from electron neutrino induced cascades. To account for this different behavior between data and simulated background under standard cuts, we have used an iterative discriminant analysis as cut level 4 (see Sec. V D) trained on a sub-sample of data (which represents the real remaining background better than the simulations) and a sub-sample of the neutralino signal. A final series of high quality cuts were applied after the discriminant analysis, bringing the remaining data sample to agree with the number of events expected from the known atmospheric neutrino flux, as shown in Fig. 2 and Table I. Note that the atmospheric neutrino curve and the data curve in Fig. 2 join and follow each other in the last two steps of the cuts applied within the level 5 filter. The following subsections give a more detailed description of the variables used and the cuts applied at each filter level.

\section{A. Filter level 1}

In a first stage, a simple and computationally fast filter based on fitting a line to the time pattern of the events was applied to the data sample in order to reject obvious downgoing tracks. This "line fit" (LF) assumes that the known space point of each hit optical module, $\vec{r}_{i}$, is related to the measured hit time, $t_{i}$, by $\vec{r}_{i}=\vec{r}_{o}+\vec{v} t_{i}$. The minimization of $\chi^{2}=\Sigma_{i}\left(\vec{r}_{i}-\vec{r}_{o}-\vec{v} t_{i}\right)^{2}$, where the index runs over all the hits in the event, leads to an explicit solution for $\vec{v}$. The zenith angle of the fitted track is readily obtained as $\cos \Theta_{\mathrm{LF}}$ $=-v_{z} /|v|$. The angular resolution of the line fit is relatively low since it does not incorporate any information about the 
TABLE I. Rejection of data, of the simulated atmospheric neutrinos and of the atmospheric-muon background samples and efficiency for the simulated neutralino signal from trigger level to filter level 5.

\begin{tabular}{lcccc}
\hline \hline Filter level & $\begin{array}{c}\text { Data } \\
130.1 \text { days } \\
\text { (events) }\end{array}$ & $\begin{array}{c}\text { Atmospheric neutrinos } \\
130.1 \text { days equivalent } \\
\text { (events) }\end{array}$ & $\begin{array}{c}\text { Atmospheric muons } \\
0.6 \text { day equivalent } \\
\text { (events) }\end{array}$ & $\begin{array}{c}\chi \bar{\chi} \rightarrow W W \\
m_{\chi}=250 \mathrm{GeV} \\
(\% \text { of trigger level) }\end{array}$ \\
\hline 0 & $1.05 \times 10^{9}$ & 4899 & $5 \times 10^{6}$ & 100 \\
$1+2$ & $2.3 \times 10^{7}$ & 2606 & $7 \times 10^{4}$ & 79 \\
3 & $1.2 \times 10^{6}$ & 472 & 2588 & 68 \\
4 & 5441 & 89 & 13 & 56 \\
5 & 14 & 16.0 & 0 & 29 \\
\hline \hline
\end{tabular}

geometry of the Cherenkov cone or about scattering of the Cherenkov photons in the ice. Still, its simplicity and computational speed makes it a very useful tool for a first assessment of the track direction and for rejection of down-going atmospheric muons [25]. The first level filter rejected obvious down-going atmospheric muons by requiring $\Theta_{\mathrm{LF}}>50^{\circ}$.

\section{B. Filter level 2}

The events that pass the level 1 filter are reconstructed using a maximum likelihood approach (ML) as described in [10]. In short, the ML technique uses an iterative process to maximize the product of the probabilities that the optical modules receive a signal at the measured times, with the track direction (zenith and azimuth angles) as free parameters. The expected time probability distributions include the scattering and absorption characteristics of the ice as well as the distance and relative orientation of the optical module with respect to the track [26].

The level 2 filter consists of two cuts: the MLreconstructed zenith angle must be larger than $80^{\circ}$ and at least three hits must be "direct." A hit is defined as direct if the time residual, $t_{\text {res }}$ (the difference between the measured time and the expected time assuming the photon was emitted from the reconstructed track and did not suffer any scattering), is small. Unscattered photons preserve the timing information. Therefore, the reconstruction of the direction of tracks with several direct hits presents a significantly better angular resolution. The number of direct hits associated with a track is the first quality requirement applied to the reconstructed data and simulated samples [24]. A residual time interval between $-10 \mathrm{~ns}$ and $25 \mathrm{~ns}$ was used to classify a hit as direct at this level.

Figure 3 shows the zenith angle distributions of simulated muon tracks from neutrinos produced in annihilation of neutralinos for the two extreme masses used in this analysis as compared to that from atmospheric neutrinos after filter level 2. The corresponding curve for data and simulated atmospheric muons is included in Fig. 1. The combined effect of these two filters on the data is a rejection of $98 \%$, as shown in Table I. The efficiencies with respect to trigger level of both level 1 and level 2 filters for simulated neutralino signal are shown in Fig. 4, for different neutralino masses and the two extreme annihilation channels used.

Filters 1 and 2 are applied in an initial data reduction common to the different subsequent analyses of the data. The rest of the cuts described below were specifically designed for the WIMP search with the aim of identifying and rejecting misreconstructions while maximizing signal detection efficiency and background rejection [27].

\section{Filter level 3}

The angular distribution of the events is the most obvious difference between the predicted neutralino signal and both the atmospheric neutrino flux and the atmospheric muon background. Neutrinos from neutralino annihilations in the center of the Earth would be concentrated in a narrow cone close to the vertical direction, while atmospheric neutrinos are distributed isotropically. The level 3 filter further restricted the ML-reconstructed zenith angle to be larger than $140^{\circ}$, placed a cut on the total number of hit modules in the event, $\mathrm{N}_{\mathrm{ch}}>10$, and on the summed hit probability of the modules with a signal, $\mathrm{P}_{\text {hit }}>0.23$. The number of hits with time residuals between $-10 \mathrm{~ns}$ and $25 \mathrm{~ns}$ was required to be larger than 4 and the number of hits with residuals between

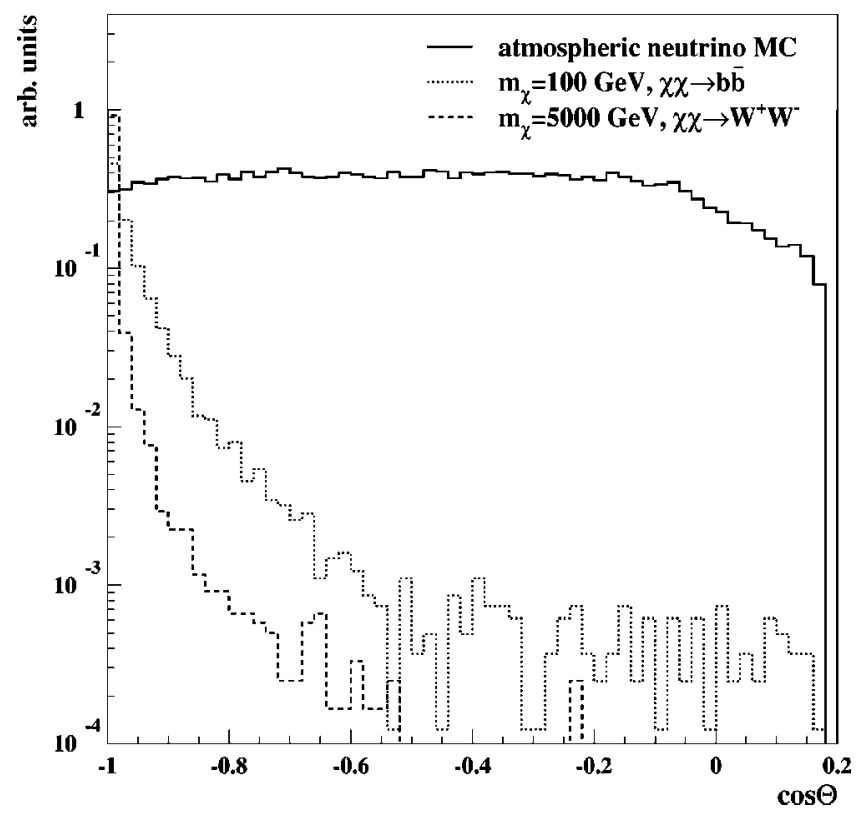

FIG. 3. Angular distribution of muons from atmospheric neutrinos and from the annihilation of neutralinos after filter level 2. The two extreme neutralino masses and annihilation channels considered in this paper are shown. The relative normalization is arbitrary. 


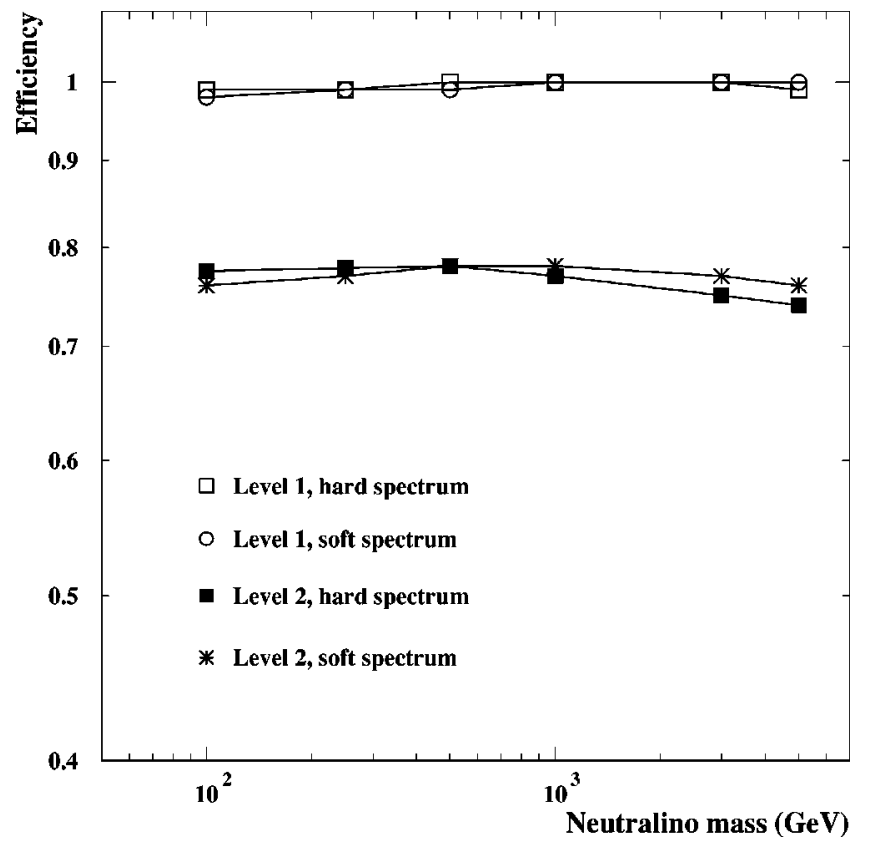

FIG. 4. Efficiencies relative to trigger level at filter levels 1 and 2 as a function of the neutralino mass.

$-15 \mathrm{~ns}$ and $75 \mathrm{~ns}$ to be larger than 5. At this stage the possible correlations between the variables are ignored, and the cuts applied to each of them individually. Table I shows the efficiency and rejection power at this cut level. Only 5 $\times 10^{-4}$ of the simulated atmospheric muon background survive this level, compared with $68 \%$ of the simulated neutralino signal and $10 \%$ of the atmospheric neutrinos.

\section{Filter level 4: iterative discriminant analysis}

To account for possible correlations between the variables and to perform a multidimensional cut in parameter space, the next filter level was based on an iterative non-linear discriminant analysis, using the IDA program [28]. Given a set of $n$ variables, the program builds the "event vector" $\mathbf{x}^{k}$ $=\left(x_{1}, \ldots, x_{n}, x_{1}^{2}, x_{1} x_{2}, \ldots, x_{1} x_{n}, x_{2}^{2}, x_{2} x_{3}, \ldots, x_{n}^{2}\right)$, where $x_{i}$ is the value of variable $i$ in event $k$. A class of events, the signal or background sample, is characterized by their mean vector $\left\langle\mathbf{x}_{s}\right\rangle$ or $\left\langle\mathbf{x}_{b}\right\rangle$, and the mean difference between the samples is given by the vector $\Delta \mu=\left\langle\mathbf{x}_{s}\right\rangle-\left\langle\mathbf{x}_{b}\right\rangle$. The spread of the variables is contained in the variance vectors, $\mu_{s}^{k}$ $=\mathbf{x}^{k}-\left\langle\mathbf{x}_{s}\right\rangle$ and $\mu_{b}^{k}=\mathbf{x}^{k}-\left\langle\mathbf{x}_{b}\right\rangle$, which are used to define a variance matrix for each class, $V^{s, b}=\Sigma_{k}{ }^{N_{\text {evts }}} \mu^{\mathbf{k}}{ }_{s, b}\left(\mu^{\mathbf{k}}{ }_{s, b}\right)^{\mathrm{T}}$, where $N_{\text {evts }}$ is the number of events in the signal or background samples and $\mathrm{T}$ denotes the transpose. The problem of separating signal from background is transformed into the problem of finding a hyperplane in event vector space which gives minimum local variance for each class and maximum separation between classes. This is translated into the requirement that the ratio $R=\left(\mathbf{a}^{\mathrm{T}} \Delta \mu\right)^{2} / \mathbf{a}^{\mathrm{T}} V \mathbf{a}$ should be maximal, where here the variance matrix $V$ is the sum of the variance matrices for signal and background and $\mathbf{a}$ is a vector of coefficients to be determined by training the program on a signal and a background sample. A target signal efficiency and background rejection factor are chosen beforehand. The coefficients a are determined in an iterative process carried out until the specified rejection factor is achieved or a predefined number of iterations reached. The coefficients found in this way are used to select events from the signal region in the multidimensional parameter space: each event is characterized by the scalar $D=\mathbf{a}^{\mathrm{T}} \mathbf{x}$ and a cut on $D$ serves as the selection criterion.

Eight variables were used in the training of the discriminant analysis program and in the subsequent cuts: the velocity of the line fit, the number of direct hits, the number of modules hit, the number of modules hit in the string with the largest number of hits, the number of detector layers with a hit, ${ }^{1}$ the extension of the event along the three coordinate axes, the average hit probability and the probability that the event time pattern is compatible with that expected from a vertical up-going muon. This set of variables includes combined information from the fit track parameters as well as the general spatial and temporal topology of the event.

Since to a first approximation the data consist of atmospheric muon background, seven days of data, evenly distributed along the year, were used as the background training sample. For the signal training sample, muons from the simulations of $250 \mathrm{GeV}$ neutralinos annihilating into a hard spectrum were used. The combination of a relatively low neutralino mass and annihilation into the hard channel was chosen as giving a "typical" muon spectrum. The target signal efficiency was set to $98 \%$ per iteration and the target global background rejection to 1000 . The stopping criterion was set to 9 iterations, based on the fact that further loops would reduce the number of events in the training sample to a too low number to be representative of the whole data set. The rejection of background achieved was 220 with respect to cut level 3 since the nine loops were exhausted before reaching the desired rejection. The overall signal efficiency attainable after the training process is then $(0.98)^{9}=0.83$. The effect of the discriminant analysis event selection is shown in Table I. It indeed achieves the expected signal efficiency, retaining $82 \%$ of the signal with respect to the previous cut level. The discrepancy of the expected number of atmospheric neutrinos and the number of remaining data events at this level indicates that the data sample is still contaminated by poorly reconstructed down-going muons. A last cut level was therefore developed to improve the rejection of the remaining misreconstructed events and select the truly up-going tracks.

\section{E. Filter level 5: final event selection}

The remaining events after the discriminant analysis with a zenith angle larger than $165^{\circ}$ were passed through the following series of cuts. The length spanned by the direct hits when projected along the track direction was required to be at least $110 \mathrm{~m}$, and the vertical length containing all hits was required to be at least $170 \mathrm{~m}$. The $z$ component of the center

\footnotetext{
${ }^{1}$ The detector was divided in eight horizontal layers of $65 \mathrm{~m}$ depth.
} 


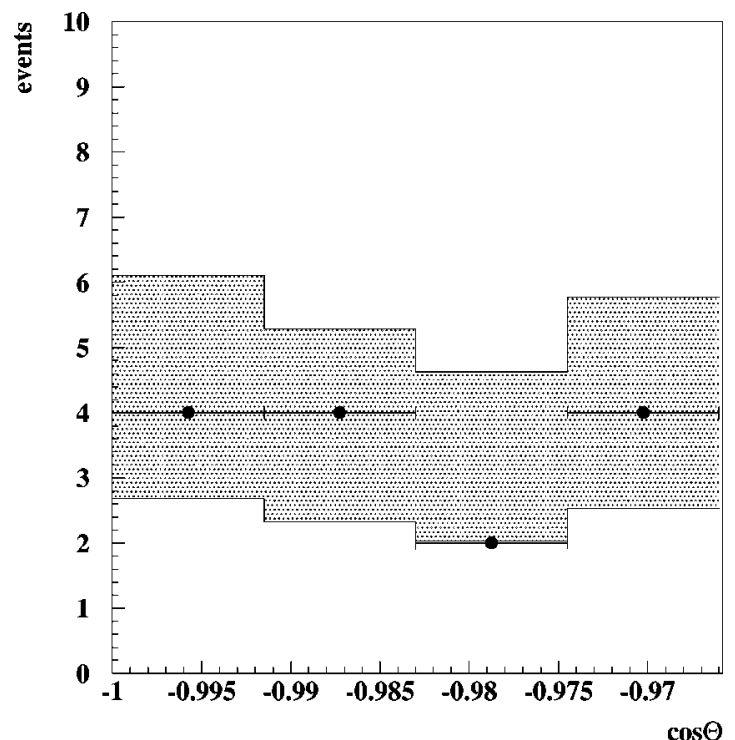

FIG. 5. Angular distribution of the remaining data events (dots) and simulated atmospheric neutrino events (shaded area) at filter level 5. The angular range shown is between $165^{\circ}$ and $180^{\circ}$. The shaded area represents the total uncertainty in the expected number of events.

of gravity of the direct hits $\left(z_{\text {c.o.g. }}=\Sigma_{\mathrm{i}} z_{\mathrm{i}} / N_{\text {direct hits }}\right.$, where the sum is over all the direct hits in the event) was required to be deeper than $1590 \mathrm{~m}$, and the percentage of hits in the lower half of the detector less than $55 \%$. These cuts reject events with a spatially uneven concentration of hits, typically due to down-going atmospheric muons that pass just outside the detector or stop close to the array.

The remaining data at this level are consistent with the expected atmospheric neutrino flux. Figure 5 shows the angular distribution of the remaining 14 data events and the remaining 16.0 simulated atmospheric neutrino events. The angular range shown is for $\Theta>165^{\circ}$, the region where a possible neutralino signal is expected to be concentrated. No statistically significant discrepancies are found between the expected number of events and angular distributions of the atmospheric neutrino background and the data. This result is also consistent with the results on atmospheric neutrinos presented in Ref. [11].

Due to the different angular shapes of the neutralino signal for different neutralino masses (see Fig. 6 for the two extreme cases considered), we have chosen to restrict further in angle the signal region we use to extract the limit on an excess muon flux. We use angular cones that contain $90 \%$ of the signal for a given neutralino mass. The remaining data and simulated atmospheric neutrino background events for the different angular cones used are shown in Table II. The background rejection power and signal efficiency from filter level 1 to 5 are shown in Fig. 2 along with the effect on the data sample.

\section{SYSTEMATIC UNCERTAINTIES}

An essential quantity when deriving limits, as we do in the next section, is the effective volume, $V_{\text {eff }}$, of the detector.

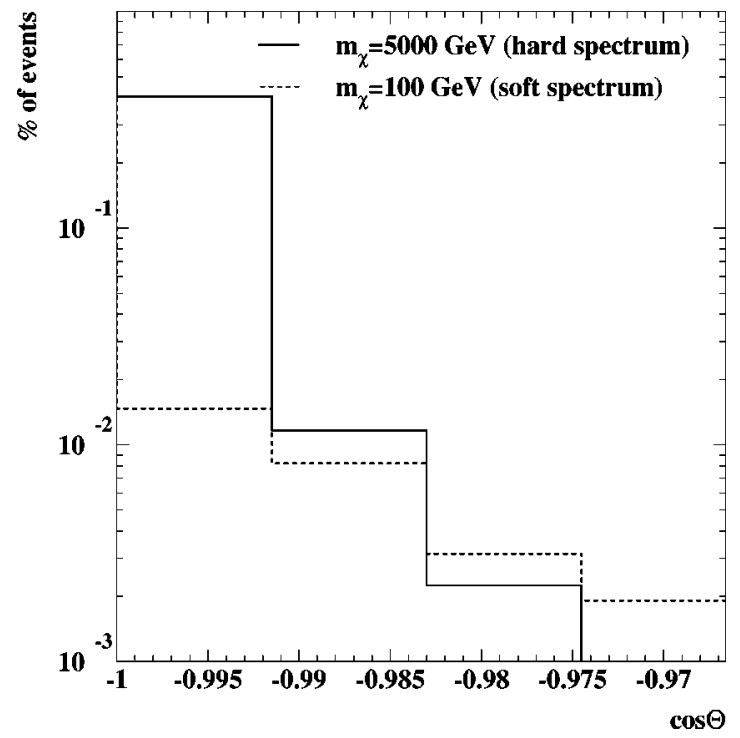

FIG. 6. Angular distribution of the remaining fraction of neutralinos at filter level 5 with respect to the trigger level from the two extreme neutralino masses studied in this paper. The angular range shown is between $165^{\circ}$ and $180^{\circ}$.

It is the measure of the efficiency to a given signal and it is defined as

$$
V_{\text {eff }}=\frac{n_{\mathrm{L} 5}}{n_{\text {gen }}} V_{\text {gen }}
$$

where $n_{\mathrm{L} 5}$ is the number of signal events after filter level 5 and $n_{\text {gen }}$ the number of events simulated in a volume $V_{\text {gen }}$

TABLE II. Number of data events, simulated atmospheric neutrino background events and the corresponding $N_{90}$ for the angular cones containing $90 \%$ of the signal for the different neutralino masses. These angular cuts are applied in addition to the level 5 filter described in Sec. V. "s" and "h" denote the soft and hard annihilation channels. The numbers in parentheses in column 5 show $N_{90}$ obtained without including systematic uncertainties.

\begin{tabular}{|c|c|c|c|c|}
\hline $\begin{array}{r}m_{\chi} \\
(\mathrm{GeV})\end{array}$ & $\begin{array}{l}\text { Angular cut } \\
\text { (deg) }\end{array}$ & $\begin{array}{c}\text { Data } \\
\text { (events) }\end{array}$ & $\begin{array}{c}\text { Atmospheric } \\
\text { neutrinos (events) }\end{array}$ & $N_{90}$ \\
\hline $100 \mathrm{~s}$ & 167.5 & 10 & 12.1 & $9.2(4.7)$ \\
\hline $100 \mathrm{~h}$ & 168.5 & 9 & 10.8 & $6.6(4.7)$ \\
\hline $250 \mathrm{~s}$ & 170.0 & 7 & 8.6 & $5.9(4.1)$ \\
\hline $\left.\begin{array}{l}250 \mathrm{~h} \\
500 \mathrm{~s}\end{array}\right\}$ & 172.0 & 5 & 6.1 & $5.6(3.9)$ \\
\hline $1000 \mathrm{~s}$ & 173.0 & 4 & 4.6 & $5.3(3.9)$ \\
\hline $500 \mathrm{~h}$ & 173.5 & 4 & 4.6 & $5.3(3.9)$ \\
\hline $\left.\begin{array}{l}1000 h \\
3000 s\end{array}\right\}$ & 174.0 & 4 & 3.9 & $5.6(4.7)$ \\
\hline $\left.\begin{array}{l}3000 h \\
5000 s \\
5000 h\end{array}\right\}$ & 174.5 & 3 & 3.9 & $4.4(3.6)$ \\
\hline
\end{tabular}




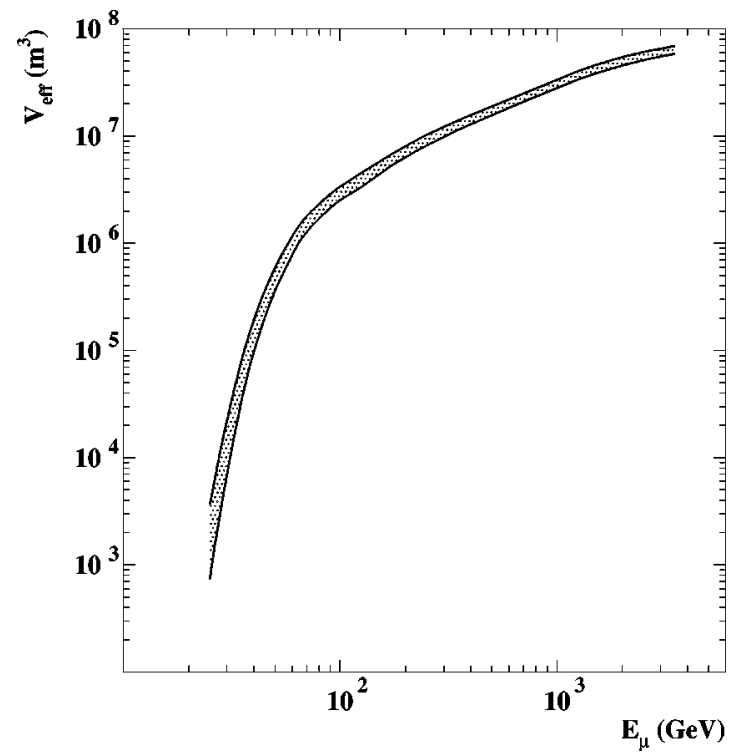

FIG. 7. Effective volume of the detector as a function of muon energy at filter level 5 .

surrounding the detector. The effective volume of AMANDA-B10 as a function of muon energy is shown in Fig. 7. Given a MSSM model producing a muon flux with a given muon energy spectrum, the effective volume of the detector for this particular signal is also calculated through Eq. (1). This is shown in Fig. 8 for the different neutralino masses used in this analysis. The shaded bands in both figures indicate the systematic uncertainty estimated as described below.

The evaluation of $V_{\text {eff }}$ is subject to experimental and theoretical systematic uncertainties present in the analysis. We have performed a detailed study of the effect of the uncertainty in several variables on the resulting effective volume by propagating variations in any of them to the final evaluation of $V_{\text {eff }}$.

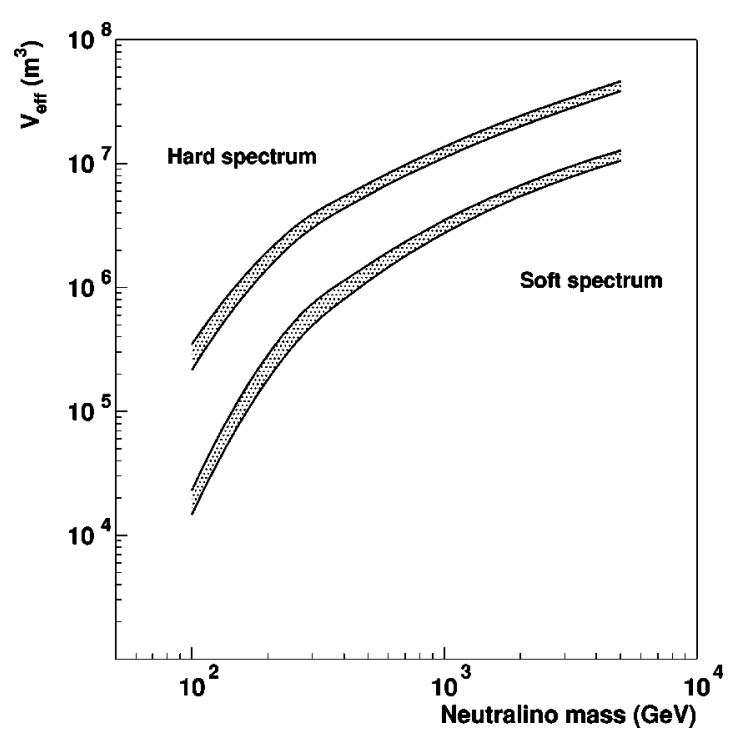

FIG. 8. Effective volumes for the neutralino signal as a function of the neutralino mass.
Measurements of the scattering and absorption lengths, $\lambda_{s}$ and $\lambda_{a}$, using pulsed and DC light sources deployed with the detector at different depths and light from an yttrium aluminum garnet (YAG) laser sent from the surface through optical fibers, have shown that these quantities exhibit a depth dependence which is correlated with dust concentration at different levels in the ice [29]. A simulation of the detector response, including layers of ice with different optical properties, has been developed and used to evaluate its effect on the results. The effects introduced are muon-energy dependent and therefore dependent on the neutralino model. The effective volumes calculated with the layered ice model are reduced between $1 \%$ and $20 \%$ with respect to the uniform ice model, except for the lower neutralino mass and soft annihilation channel $(100 \mathrm{GeV})$ where the effect reaches $50 \%$.

A further correction accounts for the uncertainties in the optical modules' total and angular sensitivities. It is known that during the process of re-freezing after deployment, air bubbles appear in the column of ice that has been melted, changing locally the scattering length of the ice and distorting the effective optical module angular sensitivity with respect to that measured in the laboratory. We have used a specific ice model for the ice in the holes that accommodates this effect. The fact that it appears after deployment and that it is not directly measurable in the laboratory makes it difficult to assess. Only by an iterative process of comparison of data and different hole-ice models can it be quantified. We estimate this effect to yield and increase of $20 \%$ in effective volume with respect to the uniform angular response model with, again, the soft annihilation channel of the lowest mass neutralino giving a stronger effect of $34 \%$. An additional $20 \%$ uncertainty on the total optical module sensitivity has been used.

The way to combine all these effects into a final estimate of the total uncertainty in $V_{\text {eff }}$ is a difficult subject, since they are not independent contributions. As described in the previous paragraphs, by varying the initial parameters used in the simulations of the detector and in the ice properties, we have obtained a range of possible values for the effective volume, which we consider as equally probable giving our current understanding of the detector. We have chosen to take the nominal $V_{\text {eff }}$ to be used in Eq. (1) as the middle value of this range. As a conservative estimate of the uncertainty we take half the width of the range of values obtained. We thus conclude that our current estimate of $V_{\text {eff }}$ is affected by a systematic uncertainty $\sigma_{V_{\text {eff }}} / V_{\text {eff }}$ between $10 \%$ and $25 \%$, depending on the neutralino mass considered, the lower mass of $100 \mathrm{GeV}$ giving the larger relative error. A similar estimate including the same effects has been made for the atmospheric neutrino Monte Carlo. In this case we estimate the uncertainty on the effective volume for atmospheric neutrinos to be $20 \%$.

Further uncertainty in the number of expected atmospheric neutrinos (column 3 in Table I) is caused by the uncertainties present in the calculation of the atmospheric neutrino flux. This is estimated to be of the order of $30 \%$ in the energy region relevant to this analysis, and originates mainly from uncertainties in the normalization of the pri- 
mary cosmic ray spectrum and in the hadronic cross sections involved [30]. This has been taken into account as an additional effect on top of the experimental uncertainty on the effective volume for atmospheric neutrinos, as described in Sec. VII B.

It has recently been shown that different muon propagation codes can produce differences in the muon flux and energy spectrum at the detector depth (see, for example, Ref. [31]). The code used in this analysis uses the Lohmann [23] parametrizations for muon energy loss, which produce results in agreement within about $10 \%$ of more recent codes [32] for muon energies up to a few of TeV. We have not included any systematics arising from the treatment of muon propagation in the ice in this analysis.

\section{RESULTS}

From the observed number of events, $n_{\text {obs }}$, and the number of expected atmospheric neutrino background events, $n_{\mathrm{B}}$, an upper limit on the signal, $N_{\beta}$, at a chosen confidence level $\beta \%$, can be obtained. We have used the unified approach for confidence belt construction [33] to calculate $90 \%$ confidence level limits. In Sec. VII B below we briefly describe a novel way of calculating limits in the presence of systematic uncertainties that we have used to obtain the final numbers presented in this paper.

\section{A. Flux limits: the standard approach}

For detectors with a fixed geometrical area $A$, it is natural to derive a muon flux limit directly through $\phi_{\mu} \leqslant \mathrm{N}_{\beta} / A \cdot t$, where $t$ is the detector live-time. However, due to the large volume of AMANDA and the lack of sharp geometrical boundaries it is the effective volume $V_{\text {eff }}$, as defined in Eq. (1), that has to be used to determine a limit on the volumetric neutrino-to-muon conversion rate, $\Gamma_{\nu \mu}$. The effective volume provides a measure of the detector efficiency since, in addition to through-going tracks, it takes into account the effect of tracks starting or stopping within the detector. A limit can then be set on $\Gamma_{\nu \mu}$, that is, on the number of muons with an energy above the detector threshold $E_{\text {thr }}$ produced by neutrino interactions per unit volume and time,

$$
\Gamma_{\nu \mu} \leqslant \frac{\mathrm{N}_{90}}{V_{\text {eff }} \cdot t}
$$

$\Gamma_{\nu \mu}$ includes all the detector threshold effects and model dependencies, as indicated below, and can be directly related to a more physically meaningful quantity, the annihilation rate, $\Gamma_{\mathrm{A}}$, of neutralinos in the center of the Earth through

$$
\begin{aligned}
\Gamma_{\nu \mu}\left(m_{\chi}\right)= & \Gamma_{\mathrm{A}} \cdot \frac{1}{4 \pi R_{\oplus}^{2}} \int_{0}^{m_{\chi}} \sum B_{\chi \bar{\chi} \rightarrow X}\left(\frac{d N_{\nu}}{d E_{\nu}}\right) \\
& \times \sigma_{\nu+N \rightarrow \mu+\ldots}\left(E_{\nu} \mid E_{\mu} \geqslant E_{\mathrm{thr}}\right) \rho_{\mathrm{N}} d E_{\nu},
\end{aligned}
$$

where the term inside the integral takes into account the production of muons through the neutrino-nucleon cross section, $\sigma_{\nu+N}$, weighted by the different branching ratios of the $\chi \bar{\chi}$ annihilation process and the corresponding neutrino energy
TABLE III. The $90 \%$ confidence level upper limits on the muon flux from neutralino annihilations in the center of the Earth, $\phi_{\mu}$, for a muon energy threshold $\geqslant 1 \mathrm{GeV}$. The last column shows the threshold-independent neutralino annihilation rate, $\Gamma_{\mathrm{A}}$. Detector systematic uncertainties have been included in the calculation of the limits. The corresponding numbers, without including uncertainties, are shown in parentheses.

\begin{tabular}{lccc}
\hline \hline$m_{\chi}(\mathrm{GeV})$ & $\begin{array}{c}\text { Annihil. } \\
\text { channel }\end{array}$ & $\begin{array}{c}\phi \mu \\
\left(\times 10^{3} \mathrm{~km}^{-2} \mathrm{yr}^{-1}\right)\end{array}$ & $\begin{array}{c}\Gamma \mathrm{A} \\
\left(\mathrm{s}^{-1}\right)\end{array}$ \\
\hline 100 & hard & $8.9(6.3)$ & $4.0(2.9) \times 10^{14}$ \\
& soft & $133.5(68.2)$ & $4.3(2.2) \times 10^{16}$ \\
250 & hard & $2.1(1.5)$ & $1.3(0.9) \times 10^{13}$ \\
& soft & $6.9(3.9)$ & $3.8(2.2) \times 10^{14}$ \\
500 & hard & $1.5(1.1)$ & $2.5(1.8) \times 10^{12}$ \\
& soft & $2.7(1.9)$ & $4.4(3.0) \times 10^{13}$ \\
1000 & hard & $1.5(1.2)$ & $6.5(5.4) \times 10^{11}$ \\
& soft & $1.8(1.4)$ & $9.2(6.8) \times 10^{12}$ \\
3000 & hard & $1.1(1.0)$ & $7.5(6.7) \times 10^{10}$ \\
& soft & $1.5(1.3)$ & $1.5(1.3) \times 10^{12}$ \\
5000 & hard & $1.1(1.0)$ & $3.2(2.8) \times 10^{10}$ \\
& soft & $1.5(1.2)$ & $7.6(6.4) \times 10^{11}$ \\
\hline \hline
\end{tabular}

spectra, $B_{\chi \bar{\chi} \rightarrow X} d N_{\nu} / d E_{\nu} \cdot \rho_{\mathrm{N}}$ is the nucleon density of the ice and $R_{\oplus}$ is the radius of the Earth. We have used a muon energy threshold of $10 \mathrm{GeV}$ in the simulations of the signal, which has been taken into account through the muon production cross section.

Equation (3) is solved for $\Gamma_{\mathrm{A}} \cdot \Gamma_{\mathrm{A}}$ depends on the MSSM model assumptions, as well as the galactic halo model used, being related to the capture rate of neutralinos in the Earth. Different neutralino models predict different capture and annihilation rates that can be probed by experimental limits set on $\Gamma_{\mathrm{A}}$. The right column of Table III shows the limits thus derived for $\Gamma_{\mathrm{A}}$. The corresponding curves are shown in Fig. 9. Quoting limits on the annihilation rate has the advantage that the detector efficiency and threshold are included through Eq. (2) and, therefore, numbers published by different experiments are directly comparable. This is not usually the case when presenting limits on muon fluxes, where at least the detector energy threshold enters in a non-trivial way and prevents direct comparison between experiments. However, since it is common in the literature to present limits on the muon flux per unit area and time, we transform below our limit on $\Gamma_{\mathrm{A}}$ into a limit on the muon flux from neutralino annihilations in the center of the Earth.

The total number of muons per unit area and time above any energy threshold $E_{\text {thr }}$ within a cone of half angle $\theta_{c}$ as a function of the annihilation rate is

$$
\begin{aligned}
\phi_{\mu}\left(E_{\mu}\right. & \left.\geqslant E_{\mathrm{thr}}, \quad \theta \geqslant \theta_{c}\right) \\
& =\frac{\Gamma_{\mathrm{A}}}{4 \pi R_{\oplus}^{2}} \int_{E_{\mathrm{thr}}}^{\infty} d E_{\mu} \int_{\theta_{c}}^{\pi} d \theta \frac{d^{2} N_{\mu}}{d E_{\mu} d \theta},
\end{aligned}
$$

where the term $d^{2} N_{\mu} / d E_{\mu} d \theta$ represents the number of muons per unit angle and energy produced from the neu- 


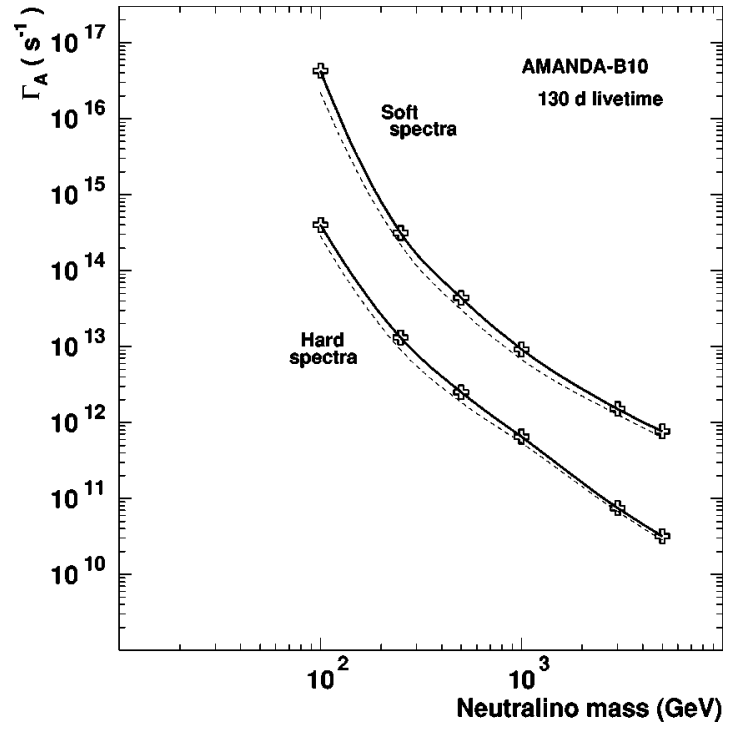

FIG. 9. $90 \%$ confidence level upper limits on the neutralino annihilation rate, $\Gamma_{\mathrm{A}}$, in the center of the Earth as a function of the neutralino mass and for the two extreme annihilation channels considered in the analysis. The dashed lines indicate the limits obtained without including systematic uncertainties and correspond to the numbers in parentheses in Table III. The symbols indicate the masses used in the analysis. Lines are to guide the eye.

tralino annihilations, and includes all the MSSM model dependencies for neutrino production from neutralino annihilation and the neutrino-nucleon interaction kinematics, as well as muon energy losses from the production point to the detector. The upper limits on the annihilation rate are thus converted to a limit on the neutralino-induced muon flux at any depth and above any chosen energy threshold and angular aperture. The $90 \%$ confidence level upper limits on the annihilation rate and the muon flux at an energy threshold of 1 $\mathrm{GeV}$ derived using Eqs. (2),(3) and (4) are shown in parentheses in Table III. The fluxes have been corrected for the inefficiency introduced by using angular cones that include $90 \%$ of the signal, so the numbers presented represent the limit on the total muon flux for each neutralino model. The threshold of $1 \mathrm{GeV}$ has been chosen to be able to compare with published limits by other experiments that have similar muon thresholds (see Sec. VIII).

\section{B. Evaluation of the limits including systematic uncertainties}

However, the best limits an experiment can set are affected by the systematic uncertainties entering the analysis. Including the known theoretical and experimental systematic uncertainties in the calculation of a flux limit is not straightforward, and often overlooked in the literature. A precise evaluation of a limit should involve the incorporation of both the uncertainties in the background counts, $\sigma_{\mathrm{b}}$, and in the effective volume, $\sigma_{\mathrm{V}}$. An additional caveat arises since the uncertainty in the effective volume introduces in turn an additional uncertainty in the expected number of background events, on top of the $30 \%$ uncertainty used in the background

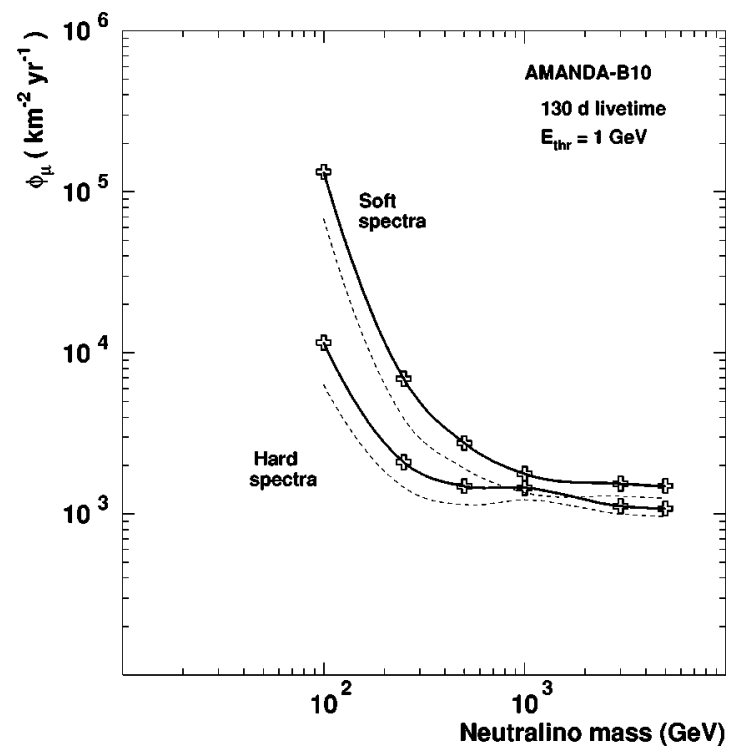

FIG. 10. 90\% confidence level upper limits on the muon flux at the surface of the Earth, $\phi_{\mu}$, as a function of the neutralino mass and for the two extreme annihilation channels considered in the analysis. The dashed lines indicate the limits obtained without including systematic uncertainties and correspond to the numbers in parentheses in Table III. The symbols indicate the masses used in the analysis. Lines are to guide the eye.

neutrino flux $\sigma_{\mathrm{b}}$. A proper implementation of the systematics in the calculation of a limit should take this correlation into account.

One approach to incorporate systematic uncertainties into an upper limit has been proposed in Ref. [34]. We have developed a similar method suited to our specific case which includes the systematic uncertainty in $V_{\text {eff }}$ in the calculation of $N_{90}$ used in Eq. (2). The method is a modified Neymantype confidence belt construction [35]. The confidence belt for a desired confidence level $\beta$ is constructed in the usual way by integrating the Poisson distribution with mean $n_{\text {tot }}$ $=n_{\mathrm{S}}+n_{\mathrm{B}}$ so as to include a $\beta \%$ probability content. But the number of events for signal and background, $n_{\mathrm{S}}$ and $n_{\mathrm{B}}$, are taken themselves to be random variables obtained from Gaussian distributions with means equal to the actual number of signal and background events observed and widths corresponding to the systematic uncertainties in signal and background.

Given an experimentally observed number of events, $N_{\text {exp }}$, the $90 \%$ confidence level limit on the number of signal events is obtained by simply inverting the calculated $N_{90}\left(n_{\text {tot }}\right)$ at the corresponding $n_{\text {tot }}=N_{\exp }$ value. In this way the different uncertainties for signal and background and the correlation between them are included naturally.

In summary, the inclusion of our present systematic uncertainties in the flux limit calculation yields results which are weakened between $\sim 10 \%$ and $\sim 40 \%$ (practically a factor of 2 for the soft channel of $m_{\chi}=100 \mathrm{GeV}$ ) with respect to those obtained using $N_{90}$ calculated without systematics. The effect is dependent on the WIMP mass, and it reflects the better sensitivity of AMANDA for higher neutrino energies. Figures 9 and 10 show the $90 \%$ confidence level limit on the 
neutralino annihilation rate and the corresponding limit on the resulting muon flux for a muon threshold of $1 \mathrm{GeV}$ for the hard and soft annihilation channels considered in the analysis. The symbols show the particular neutralino masses used in the simulation. The lines are to guide the eye and they show the limits obtained, including systematic uncertainties (solid line). The dashed lines, included for comparison, show the values obtained using the Neyman construction with the unified ordering scheme without including uncertainties. Table III summarizes the corresponding numbers.

\section{Effect of neutrino oscillations}

To account for neutrino oscillations among the different flavors, the atmospheric neutrino spectrum should be weighted by a factor $W\left(E_{\nu}\right)$, which includes the probability that a muon neutrino has oscillated into another flavor in its way through the Earth to the detector. For the purpose of illustration consider a two-flavor oscillation scenario between $\nu_{\mu}$ and $\nu_{\tau}$. Then $W\left(E_{\nu}\right)=1$ $-\sin ^{2}(2 \theta) \sin ^{2}\left[1.27 \Delta m^{2}\left(\mathrm{eV}^{2}\right) D_{\oplus}(\mathrm{km}) / E_{\nu}(\mathrm{GeV})\right]$, where $D_{\oplus}$ is the diameter of the Earth, $\theta$ the mixing angle and $\Delta m^{2}$ the difference of the squares of the flavor masses. Note that the effect depends strongly on the neutrino energy and it is negligible in the high energy tail of the atmospheric spectrum since the oscillation length is then much larger than the Earth's diameter. If we choose $\sin ^{2}(2 \theta)=1$ and $\Delta m^{2}$ $=2.5 \times 10^{-3} \mathrm{eV}^{2}$ based on the results obtained in Ref. [36], the number of expected atmospheric neutrino events is reduced between $5 \%$ and $10 \%$, depending on the angular cone considered. This would weaken the limits by about the same amount.

The effect of neutrino oscillations on the possible WIMP signal is model dependent and has been estimated in Refs. [37] and [38]. However the authors reach different conclusions on the direction of the effect: up to a factor of two in increased muon flux in Ref. [37] and a reduction of about $25 \%$ in Ref. [38] for a neutralino mass of $100 \mathrm{GeV}$. For higher neutralino masses both authors predict a less pronounced effect, which becomes negligible for the higher masses considered in [37] $\left(m_{\chi}>300 \mathrm{GeV}\right)$. We have not included any oscillation effect on the neutrinos from the WIMP signals considered in this paper.

\section{COMPARISON WITH OTHER EXPERIMENTS AND THEORETICAL MODELS}

Searches for a neutrino signal from WIMP annihilation in the center of the Earth have been performed by MACRO, Baikal, Baksan, and Super-Kamiokande.

In Fig. 11 the results of Baksan [40], MACRO [41] and Super-Kamiokande [42] are shown along with the limits from AMANDA obtained in the previous section and theoretical predictions of the MSSM as a function of WIMP mass. In order to be able to compare with the other experiments, the Super-Kamiokande limits have been scaled by a factor $1 / 0.9$ to represent total flux limits, instead of limits based on angular cones, including $90 \%$ of the signal as origi-

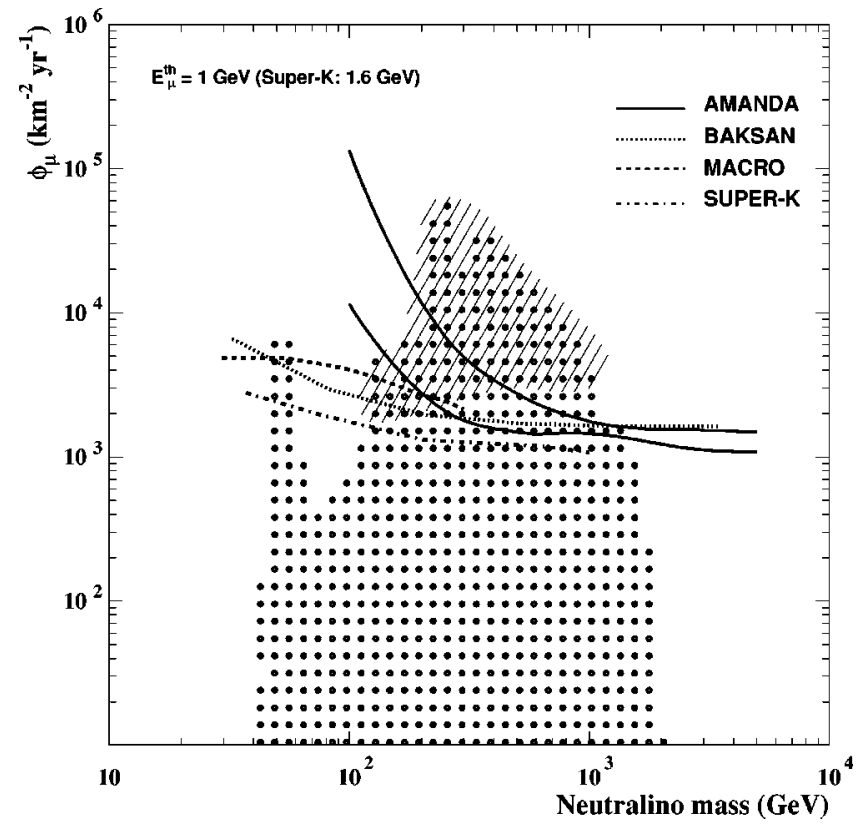

FIG. 11. The AMANDA limits on the muon flux from neutralino annihilations from Fig. 10 compared with published limits from MACRO, Baksan and Super-Kamiokande. The dots represent model predictions from the MSSM, calculated with the DarkSUSY package [39]. The dashed area shows the models disfavored by direct searches from the DAMA collaboration as calculated in [45].

nally presented in Ref. [42]. The 90\% confidence level muon flux limits for a muon energy threshold of $10 \mathrm{GeV}$ published by the Baikal collaboration range between $0.63 \times 10^{4} \mathrm{~km}^{-2} \mathrm{yr}^{-1}$ for a zenith half cone of $15^{\circ}$ and $0.54 \times 10^{4} \mathrm{~km}^{-2} \mathrm{yr}^{-1}$ for a zenith half cone of $5^{\circ}$ (Ref. [43]). Since these results are not presented as a function of WIMP mass, and are quoted at a slightly higher muon energy threshold, we have not included them in the figure but we mention them here for completeness.

Each point in the figure represents a flux obtained with a particular combination of MSSM parameters, following Ref. [44]. The original 64 free parameters of the general MSSM have been reduced to seven by the standard assumptions about the behavior of the theory at the grand unified scale and about the supersymmetry breaking parameters in the $s$-fermion sector. The independent parameters left are the Higgsino mass parameter $\mu$, the ratio of the Higgs vacuum expectation values $\tan \beta$, the gaugino mass parameter $M_{2}$, the mass $m_{\mathrm{A}}$ of the $C P$-odd Higgs boson and the quantities $m_{\mathrm{o}}, A_{\mathrm{t}}$ and $A_{\mathrm{b}}$ from the ansatz on the scale of supersymmetry breaking. These parameters were varied in the following ranges: $\quad-5000 \leqslant \mu \leqslant 5000 \mathrm{GeV}, \quad-5000 \leqslant M_{2}$ $\leqslant 5000 \mathrm{GeV}, 1.2 \leqslant \tan \beta \leqslant 50, m_{\mathrm{A}} \leqslant 1000 \mathrm{GeV}, 100 \leqslant m_{\mathrm{o}}$ $\leqslant 3000 \mathrm{GeV}, \quad-3 m_{\mathrm{o}} \leqslant A_{\mathrm{b}} \leqslant 3 m_{\mathrm{o}}$ and $-3 m_{\mathrm{o}} \leqslant \mathrm{A}_{\mathrm{t}} \leqslant 3 m_{\mathrm{o}}$. Models based on parameters already excluded by accelerator limits are not shown, and the figure is restricted to those models which give cosmologically interesting neutralino relic densities, $0.025 \leq \Omega_{\chi} h^{2}<0.5$. A local dark matter density of $0.3 \mathrm{GeV} / \mathrm{cm}^{3}$ has been assumed. Theoretical predictions for high mass neutralino models lie below the scale of 
the plot, since in this case the number density of neutralinos falls down rapidly if the dark matter density is kept fixed.

A complementary way to search for neutralinos is by measuring the nuclear recoil in elastic neutralino-nucleus collisions on an adequate target material [2]. Experiments using this direct detection technique set limits on the neutralino-nucleon cross section as a function of neutralino mass. The same scan over MSSM parameter space used to generate the theoretical points in Fig. 11 can be used to identify parameter combinations that are accessible by direct searches. There is not, however, a one-to-one correspondence between the results of the direct detection searches and the expected neutrino flux from the models probed, so comparisons with the results of indirect searches have to be performed with care. We have indicated the models disfavored by the DAMA Collaboration [45] by the dashed area in the figure, which has to be taken as an approximate region in view of the mentioned difficulties in comparing both types of detection techniques. We note that the models that yield high muon fluxes, and that are disfavored by both current results from direct searches and by the limits shown in the figure, have in common a low value of the $\mathrm{H}_{2}^{0}$ mass, around $92 \mathrm{GeV}$.

\section{SUMMARY}

We have performed a search for a statistically significant excess of vertically up-going muons with the AMANDA neutrino detector as a signature for neutralino annihilation in the center of the Earth. Limits on the neutralino annihilation rate have been derived from the non-observation of a signal excess over the predicted atmospheric neutrino background. We have included the effect of the detector systematic uncertainties and the theoretical uncertainty in the expected number of background events in the derivation of the limits, presenting in this way realistic limit values.

A comparison with the results of MACRO, Super-K and Baksan, as well as with theoretical expectations from the MSSM, is presented. AMANDA, with only 130.1 days of effective exposure in 1997, has reached a sensitivity in the high neutralino mass $(>500 \mathrm{GeV})$ region comparable to that achieved by detectors with much longer live-times.

\section{ACKNOWLEDGMENTS}

AMANDA is supported by the following agencies: The U.S. National Science Foundation, the University of Wisconsin Alumni Research Foundation, the U.S. Department of Energy, the U.S. National Energy Research Scientific Computing Center, the Swedish Research Council, the Swedish Polar Research Council, the Knut and Allice Wallenberg Foundation (Sweden) and the German Federal Ministry of Education and Research. D. F. Cowen acknowledges the support of the NSF CAREER program. C. P. de los Heros acknowledges support from the EU 4th framework of Training and Mobility of Researches. P. Loaiza was supported by the Swedish STINT program. We acknowledge the invaluable support of the Amundsen-Scott South Pole station personnel. We are thankful to I. F. M. Albuquerque and W. Chinowsky for their careful reading of the manuscript and valuable comments.
[1] L. Bergström, Rep. Prog. Phys. 63, 793 (2000).

[2] G. Jungman, M. Kamionkowski, and K. Griest Phys. Rep. 267, 195 (1996).

[3] K. Griest and M. Kamionkowski, Phys. Rev. Lett. 64, 615 (1990).

[4] G. Abbiendi et al., Eur. Phys. J. C 14, 2 (2000); 14, 187 (2000).

[5] J. Edsjö and P. Gondolo, Phys. Rev. D 56, 1879 (1997).

[6] W.H. Press and D.N. Spergel, Astrophys. J. 296, 679 (1985).

[7] K. Freese, Phys. Lett. 167B, 295 (1986); T. Gaisser, G. Steigman, and S. Tilav, Phys. Rev. D 34, 2206 (1986).

[8] A. Gould, Astrophys. J. 328, 919 (1988).

[9] J.L. Feng, K.T. Matchev, and F. Wilczek, Phys. Rev. D 63, 045024 (2001).

[10] E. Andrés et al., Astropart. Phys. 13, 1 (2000).

[11] E. Andrés et al., Nature (London) 410, 411 (2001); J. Ahrens et al., Phys. Rev. D 66, 012005 (2002).

[12] R. Wischnewski, et al., Proceedings of the XVII International Cosmic Ray Conference (ICRC), Hamburg, Germany, p. 1105; S. Barwick, et al., ibid., p. 1101.

[13] J. Edsjö, Ph.D. thesis. Uppsala University, 1997, hep-ph/9704384; L. Bergström, J. Edsjö, and P. Gondolo, Phys. Rev. D 58, 103519 (1998).

[14] T. Sjöstrand Comput. Phys. Commun. 82, 74 (1994).

[15] P. Lipari, Astropart. Phys. 1, 195 (1993).
[16] R. Ghandi et al., Astropart. Phys. 5, 81 (1996).

[17] H.L. Lai et al., Phys. Rev. D 51, 4763 (1995).

[18] E. Dalberg, Ph.D. thesis, Stockholm University, 1999, ISBN 91-7265-024-9.

[19] S.N. Boziev et al., INR preprint P-0630, Moscow, 1989.

[20] T.K. Gaisser, Cosmic Rays and Particle Physics (Cambridge University Press, Cambridge, England, 1990).

[21] D. Heck et al., FZKA report 6019, 1998, http://ik1au1.fzk.de/ heck/corsika/

[22] Particle Data Group, D. Groom et al., Eur. Phys. J. C 15, 1 (2000).

[23] W. Lohmann et al., CERN Yellow Report CERN-EP/85-03, 1985.

[24] G. Hill et al., in Proceedings of the XVI International Cosmic Ray Conference (ICRC), Salt Lake City, Utah, 1999, HE.6.3.02.

[25] V.J. Stenger, University of Hawaii preprint, HDC-1-90 (1990).

[26] C. Wiebusch, in Proceedings of the International Workshop on Simulations and Analysis Methods for Large Neutrino Telescopes, Zeuthen, Germany, 1998, edited by C. Spiering (DESY-PROC-1999-01, 1999), p. 302.

[27] P. Loaiza, Licentiat thesis, Uppsala University, 2000.

[28] T.G.M. Malmgren, Comput. Phys. Commun. 106, 230 (1997).

[29] K. Woschnagg, in Proceedings of the XVI International Cosmic Ray Conference (ICRC), Salt Lake City, Utah, 1999, HE. 4. 1. 15. 
[30] T. Gaisser, et al., in Proceedings of the XVII International Cosmic Ray Conference (ICRC), Hamburg, Germany, 2001, p. 1643.

[31] I. Sokalski et al., Phys. Rev. D 64, 074015 (2001).

[32] D. Chirkin and W. Rhode, in Proceedings of the XVII International Cosmic Ray Conference (ICRC), Hamburg, Germany, 2001, p. 1017.

[33] A. Stuart and J. K. Ord, Kendall's Advanced Theory of Statistics (Oxford University Press, New York, 1991), Vol. 2; G.J. Feldman and R.D. Cousins, Phys. Rev. D 57, 3873 (1998).

[34] R.D. Cousins and V.L. Highland, Nucl. Phys. A320, 331 (1992).

[35] J. Conrad et al., in Proceedings of the Workshop on Advanced Statistical Techniques in Particle Physics, Durham, UK, 2002, hep-ex/0202013.

[36] J. Kameda et al., in Proceedings of the XVII International Cosmic Ray Conference (ICRC), Hamburg, Germany, 2001, p. 1057.

[37] M. Kowalski, Phys. Lett. B 511, 119 (2001).

[38] N. Fornengo, in Proceedings of the Third International Conference on Dark Matter in Astro and Particle Physics, Heidelberg,
Germany, 2000. Also hep-ph/0011030.

[39] P. Gondolo, et al. in Proceedings of the Third International Workshop on Identification of Dark Matter (IDM2000), York, UK, 2000, edited by N.J.C. Spooner and V. Kudryavtsev (World Scientific, Singapore, 2001), p. 318; P. Gondolo, astro-ph/0012234; http://www.physto.se/ edsjo/darksusy/.

[40] M. Boliev et al., in Proceedings of Dark Matter in Astro and Particle Physics, 1997, edited by H.V. Klapdor-Kleingrothaus and Y. Ramachers (World Scientific, Singapore, 1997), p. 711; see also O. Suvorova, hep-ph/9911415.

[41] M. Ambrosio et al., Phys. Rev. D 60, 082002 (1999).

[42] A. Habig, et al., Proceedings of the XVII International Cosmic Ray Conference (ICRC), Hamburg, Germany, 2001, p. 1558; hep-ex/0106024.

[43] V.A. Balkanov et al., Proceedings of Neutrino 2000, Sudbury, Canada, 2000; Nucl. Phys. B91, 438 (2001).

[44] L. Bergström, J. Edsjö, and P. Gondolo, Phys. Rev. D 55, 1765 (1997).

[45] L. Bergström, J. Edsjö, and P. Gondolo, Phys. Rev. D 58, 103519 (1998). 